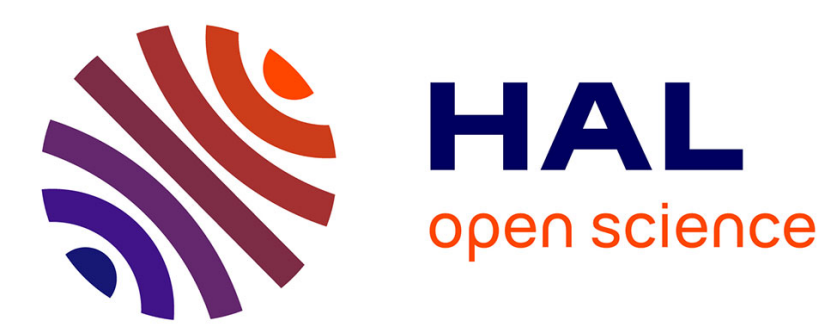

\title{
Impact of Topology and Shadowing on the Outage Probability of Cellular Networks
}

\author{
Jean-Marc Kélif, Marceau Coupechoux
}

\section{To cite this version:}

Jean-Marc Kélif, Marceau Coupechoux. Impact of Topology and Shadowing on the Outage Probability of Cellular Networks. IEEE International Conference on Communications, ICC, Jun 2009, Dresden, Germany. pp.1-6, 10.1109/ICC.2009.5199067 . hal-00694503

\section{HAL Id: hal-00694503 https://hal-imt.archives-ouvertes.fr/hal-00694503}

Submitted on 4 May 2012

HAL is a multi-disciplinary open access archive for the deposit and dissemination of scientific research documents, whether they are published or not. The documents may come from teaching and research institutions in France or abroad, or from public or private research centers.
L'archive ouverte pluridisciplinaire HAL, est destinée au dépôt et à la diffusion de documents scientifiques de niveau recherche, publiés ou non, émanant des établissements d'enseignement et de recherche français ou étrangers, des laboratoires publics ou privés. 


\section{Impact of Topology and Shadowing on the Outage Probability of Cellular Networks}

\author{
Jean-Marc Kelif \\ Orange Labs \\ Issy-Les-Moulineaux, France \\ jeanmarc.kelif@orange-ftgroup.com
}

\author{
Marceau Coupechoux \\ TELECOM ParisTech \& CNRS LTCI \\ 46, rue Barrault, Paris, France \\ marceau.coupechoux@telecom-paristech.fr
}

\begin{abstract}
This paper proposes an analytical study of the shadowing impact on the outage probability in cellular radio networks. We establish that the downlink other-cell interference factor, $f$, which is defined here as the ratio of outer cell received power to the inner cell received power, plays a fundamental role in the outage probability. From $f$, we are able to derive the outage probability of a mobile station (MS) initiating a new call. Taking into account the shadowing, $f$ is expressed as a lognormal random variable. Analytical expressions of the interference factor's mean $m_{f}$ and standard deviation $s_{f}$ are provided in this paper. These expressions depend on the topology of the network characterized by a $G$ factor. We show that shadowing increases the outage probability, and using our analytical method, we are able to quantify this impact. However, we establish that the network topology, or correlated received powers, may limit this increase.
\end{abstract}

\section{INTRODUCTION}

The estimation of cellular networks capacity mainly depends on the characterization of interference. As downlink is often the limited link w.r.t. capacity, we focus on this direction throughout this paper, although the proposed framework can easily be extended to the uplink. In cellular networks, an important parameter for this characterization is the other-cell interference factor $f$ (OCIF). The precise knowledge of the OCIF allows the derivation of outage probabilities, capacity evaluation and then, the definition of Call Admission Control mechanisms. In this paper, we define OCIF as the ratio of total other-cell received power to the total inner-cell received power. Working on the uplink, [14] derived the distribution function of a ratio of path-losses, which is essential for the evaluation of external interference. For that, authors approximate the hexagonal cell with a disk of same area.

On the downlink, [1] [2] aimed at computing an average OCIF over the cell by numerical integration in hexagonal networks. In [13], other-cell interference is given as a function of the distance to the base station (BS) thanks to MonteCarlo simulations. Chan and Hanly [12] precisely approximate the distribution of the other-cell interference. They however provide formulas that are difficult to handle in practice. Considering random networks, Baccelli et al. [11] provide spatial blocking probabilities in random networks by using gaussian approximation. Since in a real network, the power received at any point of the system depends on the local environment (terrain, buildings, trees), the shadowing has to be considered. It is generally modelled as a lognormal distributed [6] function. Reference [7] computes the outage probability in presence of multiple log-normal interferers, but assumes that all interference signals have the same statistics and mobile location influence is not studied. In this paper, we take into account correlated shadowing and the location of interferers and mobile stations in the calculation of the OCIF. Moreover, our approach gives a new lighting concerning the influence of topology.

We first introduce the model and the notations. Then, we establish the interference factor's analytical expressions, mean value and standard deviation, as a lognormal random variable (RV) according to the Fenton-Wilkinson approximation for a sum of lognormal RV [4]. We show that the environment of the system is characterized by a function $H$, and we establish its analytical expression. That function depends on the shadowing and on the topology of the network through a $\mathbf{G}$ factor. Using moreover the expressions of $f$ without shadowing established in [8] [9], we calculate analytically the outage probability by using a Gaussian approximation. We show that the shadowing increases the outage probability, and we quantify this increase. We moreover establish that the effects of correlated received powers and topology decrease the impact of shadowing.

\section{INTERFERENCE MODEL AND NOTATIONS}

We consider a cellular radio system and we focus on the downlink. BS have omni-directional antennas, so that a BS covers a single cell. If a mobile $u$ is attached to a station $b$ (or serving BS), we write $b=\psi(u)$.

The following power quantities are considered: $P_{b, u}$ is the transmitted power from station $b$ towards mobile $u$ (for user's traffic), $P_{b}=P_{c c h}+\Sigma_{u} P_{b, u}$ is the total power transmitted by station $b, P_{c c h}$ represents the amount of power used to support broadcast and common control channels, $g_{b, u}$ designates the pathgain between station $b$ and mobile $u, p_{b, u}$ is the power received at mobile $u$ from station $b$, we can write $p_{b, u}=$ $P_{b} g_{b, u}, S_{b, u}=P_{b, u} g_{b, u}$ is the useful power received at mobile $u$ from station $b$ (for traffic data); since we do not consider soft handover, we can write $S_{u}=S_{\psi(u), u}$.

We consider a reuse 1 radio system such as OFDMA or CDMA. The total amount of power experienced by a mobile station $u$ in a cellular system can be split up into several terms: useful signal $\left(S_{u}\right)$, interference and noise $\left(N_{t h}\right)$. It is common 
to split the system power into two terms: $I_{u}=I_{i n t, u}+I_{e x t, u}$, where $I_{\text {int }, u}$ is the internal (or own-cell) received power and $I_{e x t, u}$ is the external (or other-cell) interference. Notice that we made the choice of including the useful signal $S_{u}$ in $I_{i n t, u}$, and, as a consequence, it has to be distinguished from the commonly considered own-cell interference.

With the above notations, we define the interference factor in $u$, as the ratio of total power received from other BS to the total power received from the serving BS $\psi(u): f_{u}=$ $I_{e x t, u} / I_{\text {int }, u}$. The quantities $f_{u}, I_{e x t, u}$, and $I_{i n t, u}$ are location dependent and can thus be defined in any location $x$ as long as the serving BS is known.

In downlink, a coefficient $\alpha$, may be introduced to account for the lack of perfect orthogonality in the own cell. In a CDMA system $0<\alpha<1$, and in an OFDMA system $\alpha=0$.

In this paper, we use the signal to interference plus noise ratio (SINR) as the criteria of radio quality: $\gamma_{u}^{*}$ is the SINR target for the service requested by MS $u$ or required to obtain a minimum throughput in case of elastic traffic. This figure is a priori different from the SINR evaluated at mobile station $u$. However, we assume perfect power control, so $S I N R_{u}=\gamma_{u}^{*}$ for all users. With the introduced notations, the SINR experimented by $u$ can thus be derivated (see e.g. [3]):

$$
\gamma_{u}^{*}=\frac{S_{u}}{\alpha\left(I_{i n t, u}-S_{u}\right)+I_{e x t, u}+N_{t h}} .
$$

In case of OFDMA, this equation is valid per sub-carrier. We can now express $S_{u}$ as:

$$
S_{u}=\frac{\gamma_{u}^{*}}{1+\alpha \gamma_{u}^{*}} I_{i n t, u}\left(\alpha+I_{e x t, u} / I_{i n t, u}+N_{t h} / I_{i n t, u}\right) .
$$

Using relations $I_{i n t, u}=P_{b} g_{b, u}$ and $f_{u}=I_{e x t, u} / I_{i n t, u}$ we can write

$$
f_{u}=\frac{\sum_{j \neq b} P_{j} g_{j, u}}{P_{b} g_{b, u}}
$$

and the transmitted power for MS $u, P_{b, u}=S_{u} / g_{b, u}$,

$$
P_{b, u}=\frac{\gamma_{u}^{*}}{1+\alpha \gamma_{u}^{*}}\left(\alpha P_{b}+f_{u} P_{b}+N_{t h} / g_{b, u}\right) .
$$

From this relation, the output power of BS $b$ can be computed as follows:

$$
P_{b}=P_{c c h}+\sum_{u} P_{b, u}
$$

and so, according to Eq.4,

$$
P_{b}=\frac{P_{c c h}+\sum_{u} \frac{\gamma_{u}^{*}}{1+\alpha \gamma_{u}^{*}} \frac{N_{t h}}{g_{b, u}}}{1-\sum_{u} \frac{\gamma_{u}^{*}}{1+\alpha \gamma_{u}^{*}}\left(\alpha+f_{u}\right)} .
$$

Note that this equation is valid for multiple SINR targets and thus for multi-service networks since $\gamma_{u}^{*}$ is specific to user $u$. The knowledge of the interference factor is needed to analyze outage probability (see Section V) since the base station transmitting power depends on this parameter.

\section{ShadowING INFLUENCE}

\section{A. Propagation}

Considering the power $P_{j}$ transmitted by the $\mathrm{BS} j$, the power $p_{j, u}$ received by a mobile $u$ can be written:

$$
p_{j, u}=P_{j} K r_{u}^{-\eta} A
$$

$A=10^{\frac{\xi}{10}}$ represents the shadowing effect. The term A characterizes the random variations of the received power around a mean value. $\xi$ is a Normal distributed random variable $R V$, with mean 0 and standard deviation comprised between 0 and $10 \mathrm{~dB}$. The term $P_{j} K r_{u}^{-\eta}$, where $\mathrm{K}$ is a constant, represents the mean value of the received power at distance $r_{u}$ from the transmitter $\left(B S_{j}\right)$. The probability density function (PDF) of this slowly varying received power is given by

$$
p(s)=\frac{1}{a \sigma s \sqrt{\pi}} \exp -\left(\frac{\ln (s)-a m}{\sqrt{2} a \sigma}\right)^{2}
$$

where $a=\frac{\ln 10}{10}, m=\frac{1}{a} \ln \left(K P_{j} r_{u}^{-\eta}\right)$ is the (logarithmic) received mean power expressed in decibels $(\mathrm{dB})$, which is related to the path loss and $\sigma$ is the (logarithmic) standard deviation of the mean received signal due to the shadowing.

\section{B. Interference factor}

Since the interference factor is defined as $f_{u}=$ $I_{\text {ext }, u} / I_{\text {int }, u}$, we first calculate the other cell interference power due to all the BS of the network (except the serving one $b) I_{e x t, u}=\sum_{j \neq b}^{B} P_{j} g_{j, u}$ is the sum of $B$ lognormal RV. Such a sum can be approximated by another lognormal distribution [6]. Using the Fenton-Wilkinson [4] method, we aim to calculate the interference factor, for any mobile at the distance $r_{u}$ from its serving the $B S_{b}$, as a lognormal RV (mean and standard deviation). We first calculate the mean and the variance of a sum of lognormal RV. We afterwards apply the result to the sum of $B$ lognormal identically distributed RV. For the sake of simplicity, we denote $r_{u}=r$. Expressing the mean interference power received by a mobile, due to all the other base stations of the network (appendix 1) and since the ratio of two lognormal RV's is also expressed as a lognormal $\mathrm{RV}$, the interference factor is also lognormally distributed with the following mean $m_{f}$ and logarithmic variance $s_{f}$. We consider that all the standard deviations are identical $\sigma_{j}=\sigma$. Assuming that all the base stations have the same transmitting power $P_{b}=P_{j}=P$ (uniform traffic), we introduce

$$
\begin{gathered}
G(r, \eta)=\frac{\sum_{j} r_{j}^{-2 \eta}}{\left(\sum_{j} r_{j}^{-\eta}\right)^{2}}, \\
f_{0}(r, \eta)=\frac{\sum_{j} r_{j}^{-\eta}}{r^{-\eta}}, \\
H(r, \sigma)=e^{a^{2} \sigma^{2} / 2}\left(G(r, \eta)\left(e^{a^{2} \sigma^{2}}-1\right)+1\right)^{-\frac{1}{2}} .
\end{gathered}
$$

From (3) and (7), we notice that $f_{0}(r, \eta)$ represents the interference factor $f$ without shadowing. 
In this paper, we consider only omni-directional antennas. For sectorized networks, the expression of $f$ is modified to take into account antenna patterns (see [15] for more details).

1) Powers without correlation: We can express (appendix 2):

$$
m_{f}=f_{0}(r, \eta) H(r, \sigma) .
$$

The standard deviation is given by

$$
a^{2} s_{f}^{2}=2\left(a^{2} \sigma^{2}-\ln H(r, \sigma)\right) .
$$

2) Correlated powers : Introducing an average correlation coefficient $t$ and 'correlated variance' $\sigma_{g}^{2}=\sigma^{2}(1-t)$, we can express (see appendix 3):

$$
m_{f, c o r r}=f_{0}(r, \eta) H\left(r, \sigma_{g}\right) .
$$

The standard deviation is given by

$$
a^{2} s_{f, \text { corr }}^{2}=2\left(a^{2} \sigma_{g}^{2}-\ln H\left(r, \sigma_{g}\right)\right)+2 t a^{2} \sigma^{2} .
$$

We established that by introducing a 'correlated variance', the analytical expressions for independant powers and correlated powers are similar.

\section{TOPOLOGICAL ANALYSIS}

\section{A. Topological characterization}

The expression (12) means that the effect of the environment of any mobile of a cell, on the interference factor, is characterized by a function $H(r, \sigma)$. This function takes into account the shadowing and a topological $\mathbf{G}$ factor which depends on the position of the mobile and the characteristics of the network as

- the exponential pathloss parameter $\eta$, which can vary with the topography and more generally with the geographical environment as urban or country, micro or macro cells.

- the base stations positions and number.

We notice that the topological factor $\mathrm{G}$ can be rewritten (generalizing (10) and dropping r) as:

$$
G(\eta)=\frac{f_{0}(2 \eta)}{f_{0}(\eta)^{2}}
$$

The higher the standard deviation of shadowing, the higher the mean and standard deviation of the interference factor (see hereafter Eq. (17) and (18)). This increase is however bounded as we can see hereafter. In a realistic network, $\sigma$ is generally comprised between 6 and $12 \mathrm{~dB}$.

\section{B. Interference factor limits}

1) Non correlated powers: From (9), we have $0<$ $G(r, \eta)<1$ whatever $r$ and $\eta$. From (12) and (13), the interference factor's limits can be expressed as:

$$
f_{0}(r, \eta) \leq m_{f} \leq \frac{f_{0}(r, \eta)}{G(r, \eta)^{\frac{1}{2}}}
$$

and

$$
s_{f}^{2} \leq 2 \sigma^{2} .
$$

2) Correlated powers: When $t=1$, i.e. powers totally correlated, we have $H\left(r, \sigma_{g}\right)=1$ and $m_{f}=f_{0}(r, \eta)$ as if there were no shadowing. The standard deviation is given by $s_{f}^{2}=2 \sigma^{2}$.

For low standard deviations (less then 4 or $5 \mathrm{~dB}$ ), we can easily deduce from (12) and (13) a low dependency of the mean value of $f$ with $\sigma$, and the total standard deviation $s_{f}$ is very close to $\sigma$. These expressions show another interesting result. We established that $0<G<1$. Without the topological factor $\mathrm{G}(G=0)$, we have $H=e^{a^{2} \sigma^{2}}$ so that $m_{f}$ increases indefinitly. If $G=1$, then $H=1$ and $m_{f}=f$. For each given value of $\eta$, characterizing a given type of cells or environment, the topological factor $\mathrm{G}$ thus compensates the shadowing effects.

\section{OUtAge PRobabilities}

For a given number of MS per cell, $n$, outage probability, $P_{\text {out }}^{(n)}$, is the proportion of configurations, for which the needed BS output power exceeds the maximum output power: $P_{b}>$ $P_{\max }$. If noise is neglected and if we assume a single target SINR $\left(\gamma_{u}^{*}=\gamma^{*}\right.$ for all $\left.u\right)$, we deduce from Eq.6:

$$
P_{\text {out }}^{(n)}=\operatorname{Pr}\left[\sum_{u=0}^{n-1}(f(r, \eta))>\frac{1-\varphi}{\beta}-n \alpha\right],
$$

where $\varphi=P_{c c h} / P_{\max }$ and $\beta=\gamma^{*} /\left(1+\alpha \gamma^{*}\right)$.

\section{A. Outage probability without shadowing}

Without shadowing, the interference factor is a deterministic function, for each mobile location $r$. However, each mobile enters the cell at a random location. Hence, the function $\sum_{u=0}^{n-1} f_{0}(r, \eta)$ (since $f_{0}$ represents the interference factor $f$ without shadowing) is random and depends on the position of the $\mathrm{n}$ mobiles in the cell.

In order to compute these probabilities, we rely on the Central Limit theorem and use a Gaussian approximation. We can write:

$$
P_{\text {out }}^{(n)}=Q\left(\frac{\frac{1-\varphi}{\beta}-n \mu_{f_{0}}-n \alpha}{\sqrt{n} \sigma_{f_{0}}}\right),
$$

where $Q$ is the error function, $\mu_{f_{0}}$ and $\sigma_{f_{0}}$ are respectively the spatial mean and standard deviation of $f_{0}$ over the cell area.

As a consequence, we need to compute the spatial mean and standard deviation of $f_{0}$. For a mobile at the distance $r$ from its serving base station, and dropping the dependance with $\eta$, the interference factor can be written as $f_{0}(r)$ [8] [9]:

$$
f_{0}(r)=\frac{2 \pi \rho_{B S} r^{\eta}}{\eta-2}\left(2 R_{c}-r\right)^{2-\eta} .
$$

where $1 / \rho_{B S}=\pi R_{e}^{2}$ represents the area of a cell, and $2 R_{c}$ the distance between 2 neighbours base stations. We have $R_{e}=$ $R_{c} \sqrt{2 \sqrt{3} / \pi}$. So, we integrate $f_{0}(r)$ on a disk of radius $R_{e}$. As MS are uniformly distributed over the disk of radius $R_{e}$, the PDF of $r$ is: $p_{r}(t)=\frac{2 t}{R_{e}^{2}}$. 


$$
\begin{aligned}
\mu_{f_{0}}= & \frac{2 \pi \rho_{B S}}{\eta-2} \int_{0}^{R_{e}} t^{\eta}\left(2 R_{c}-t\right)^{2-\eta} \frac{2 t}{R_{e}^{2}} d t \\
= & \frac{2^{4-\eta} \pi \rho_{B S} R_{c}^{2}}{\eta^{2}-4}\left(\frac{R_{e}}{R_{c}}\right)^{\eta} \times \\
& { }_{2} F_{1}\left(\eta-2, \eta+2, \eta+3, R_{e} / 2 R_{c}\right),
\end{aligned}
$$

where ${ }_{2} F_{1}(a, b, c, z)$ is the hypergeometric function, whose integral form is given by:

$$
{ }_{2} F_{1}(a, b, c, z)=\frac{\Gamma(c)}{\Gamma(b) \Gamma(c-b)} \int_{0}^{1} \frac{t^{b-1}(1-t)^{c-b-1}}{(1-t z)^{a}} d t,
$$

and $\Gamma$ is the gamma function.

Note that for $\eta=3$, we have the simple closed formula:

$$
\mu_{f_{0}}=-2 \pi \rho_{B S} R_{c}^{2}\left(\frac{\ln (1-\nu / 2)}{\nu^{2}}+\frac{16}{\nu}+4+\frac{4 \nu}{3}+\frac{\nu^{2}}{2}\right),
$$

where $\nu=R_{e} / R_{c}$. In the same way, the variance of $f(r)$ is given by:

$$
\begin{aligned}
\sigma_{f_{0}}^{2}= & E\left[f_{0}^{2}\right]-\mu_{f_{0}}^{2} \\
E\left[f_{0}^{2}\right]= & \frac{2^{4-2 \eta}\left(2 \pi \rho_{B S} R_{c}^{2}\right)^{2}}{(\eta+1)(\eta-2)^{2}}\left(\frac{R_{e}}{R_{c}}\right)^{2 \eta} \times \\
& { }_{2} F_{1}\left(2 \eta-4,2 \eta+2,2 \eta+3, \frac{R_{e}}{2 R_{c}}\right) .
\end{aligned}
$$

\section{B. Outage probability: shadowing impact}

Considering the shadowing impact, the interference factor is a lognormal random variable function, for each mobile location $r$. Using a Gaussian approximation, the outage probability is now expressed as:

$$
P_{\text {out }}^{(n)}=Q\left(\frac{\frac{1-\varphi}{\beta}-n M_{f}-n \alpha}{\sqrt{n} S_{f}}\right),
$$

$M_{f}$ and $S_{f}$ are respectively the spatial mean and standard deviation of the lognormal random variable $f(r, \eta)$ characterized by $m_{f}$ and $s_{f}$ (Eq. 12 and 13) over the cell area.

Since the interference factor is expressed as a lognormal random variable $f$, so $\ln (f)$ is a normal random variable expressed as $N\left(\ln m_{f}, a^{2} \sigma_{f}^{2}\right)$. The mean $E[f(r)]$ and variance $\operatorname{Var}[f(r)]$ of the interference factor can be written:

$$
\begin{aligned}
E[f(r)] & =e^{\ln m_{f}+a^{2} s_{f}^{2} / 2}=m_{f} e^{a^{2} s_{f}^{2} / 2}, \\
\operatorname{Var}[f(r)] & =\left(e^{a^{2} s_{f}^{2}}-1\right) e^{2 \ln m_{f}+a^{2} s_{f}^{2}} .
\end{aligned}
$$

So we have

$$
\begin{aligned}
M_{f}(\sigma) & =\int_{0}^{R_{e}} E[f(t)] p_{r}(t) \\
& =\int_{0}^{R_{e}} f_{0}(t) H(t, \sigma) e^{a^{2} s_{f}^{2} / 2} \frac{2 t}{R_{e}^{2}} d t .
\end{aligned}
$$

In the same way, the spatial variance of $f$ is given by:

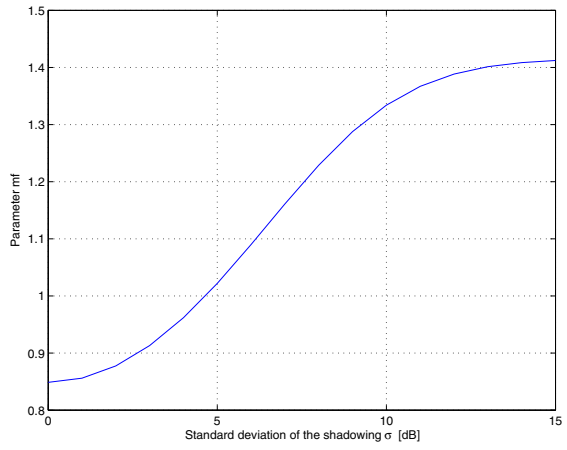

Fig. 1. Variations of $m_{f}$ as a function of $\sigma$ for $r=R_{c}$.

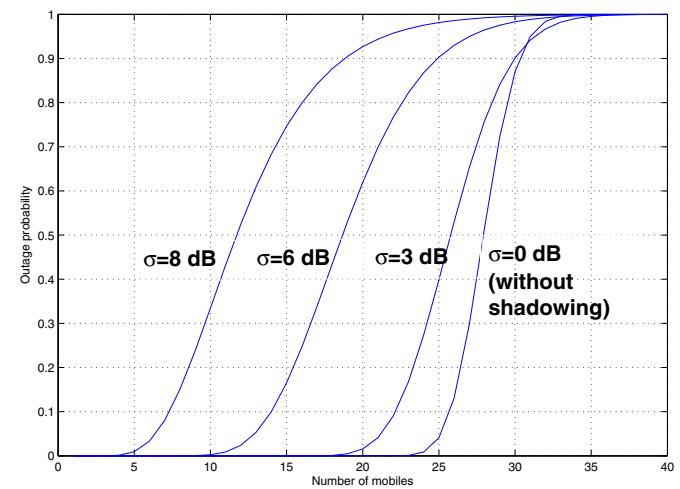

Fig. 2. Outage probability for correlated power $t=50 \%$, without shadowing $(\sigma=0)$, and with shadowing $(\sigma=3 \mathrm{~dB}, \sigma=6 \mathrm{~dB}$ and $\sigma=8 \mathrm{~dB})$, with a correlation $t=50 \%$.

$$
\begin{aligned}
S_{f}^{2}(\sigma) & =E\left[f^{2}\right]-E^{2}[f]=E\left[f^{2}\right]-M_{f}^{2}(\sigma) \\
E\left[f^{2}\right] & =\int E\left[f^{2} \mid r\right] p(r) d r \\
& =E\left[E[f(r)]^{2}+\operatorname{Var}[f(r)]\right] \\
& =\int_{0}^{R_{e}}\left(\left(f_{0}(t) H(t, \sigma)\right)^{2} e^{2 a^{2} s_{f}^{2}} \frac{2 t}{R_{e}^{2}} d t .\right.
\end{aligned}
$$

\section{Results}

Figure 1 shows that in presence of shadowing, the mean interference $m_{f}$ factor increases $(\eta=4)$. However, we observe this increase reaches a maximum value for $\sigma=15 \mathrm{~dB}$ approximately. It is due to the effect of the topology factor $\mathrm{G}$ which compensates the effect of shadowing.

Figures 2 and 3 give examples of the kind of results we are able to obtain instantaneously thanks to the formulas derived in this paper for voice service $\left(\gamma_{u}^{*}=-16 \mathrm{~dB}\right)$ and for the path-loss exponent $\eta=4$.

Figure 2 shows the outage probabilities as a function of the number of MS per cell for different values of $\sigma$ and for a correlation of $50 \%$. It allows us to easily find the capacity of the network at any given percentage of outage. It moreover 


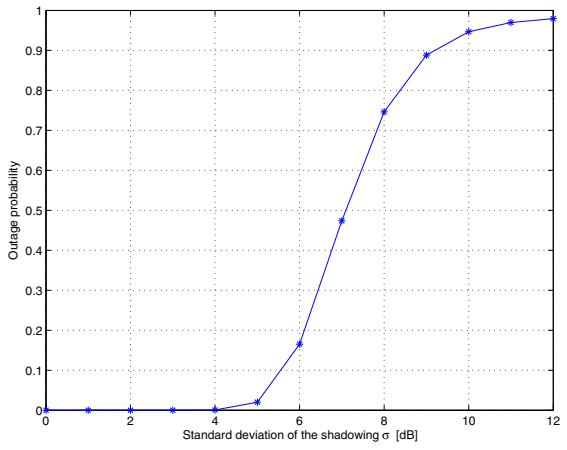

Fig. 3. Impact of the shadowing on the outage probability when there are 15 mobiles in the cell.

shows us the impact of shadowing on the capacity. As an example, for an outage probability of $10 \%$, the cell capacity is $7,14,22$, and 26 users for $\sigma=8 \mathrm{~dB}, 6 \mathrm{~dB}, 3 \mathrm{~dB}$ and without shadowing respectively.

Figure 3 focuses on the outage probability as a function of the shadowing when there are 15 mobiles per cell. Until $\sigma=5 \mathrm{~dB}$, the outage probability is quite low (less than $5 \%$ ). For higher values of $\sigma$, the outage probability increases rapidly.

The high level of outage probability observed may be due to the fact that in our analysis mobiles are not assumed belonging to the 'best serving' BS but to the closest one.

With these results, an operator would be able to analyze the outage, and to admit or reject new connections according to each specific environment of the entering MS. Indeed, the interference factor depends on the mobile location and on the environment (expressions 12 and 13), i.e. the shadowing, the exponential pathloss parameter $\eta$ (which depends on different characteristics and particularly the cell dimensions and the type of environment e.g. urban or country), the number and locations of the base stations.

\section{CONCLUSION}

Taking into account shadowing, we established the OCIF $f$ as a lognormal random variable, and expressed close form formulas of the mean $m_{f}$ and standard deviation $s_{f}$. They depend on the topology of the network through a topological factor $\mathrm{G}$, which has the effect of partially compensating shadowing. It allowed a spatial integration of $f$ leading to express analytically the outage probability. We showed that the impact of the shadowing is to increase the outage probability, and we quantified precisely this increase. Moreover, we established that correlated powers and topology effects may limit the shadowing impact. Though focused on CDMA and OFDMA networks, the proposed framework can be applied to other wireless systems, such as TDMA or ad-hoc ones.

\section{APPENDIX 1: INTERFERENCE POWER}

Each BS transmits a power $P_{j}=P$ so the power received by a mobile is characterized by a lognormal distribution $X_{j}$ as $\ln \left(X_{j}\right) \propto N\left(a m_{j}, a^{2} \sigma_{j}^{2}\right)$ and we can write $m_{j}=\frac{1}{a} \ln \left(P_{j} r_{j}^{-\eta}\right)$ (to simplify the calculation we consider $\mathrm{K}=1$ ). So the total power received by a mobile is a lognormal $\mathrm{RV} \mathrm{X}$ characterized by its mean and variance $\ln (X) \propto N\left(a m, a^{2} \sigma_{t}^{2}\right)$ and we can write: $a m=\ln \left(\sum_{j=0, j \neq b}^{B} e^{\left(\ln P_{j}-\eta \ln r_{j}+\frac{a^{2} \sigma_{j}^{2}}{2}\right)}\right)-\frac{a^{2} \sigma_{t}^{2}}{2}$ so we have since $P_{j}=P$ whatever the base station $\mathrm{j}$, considering identical $\sigma_{j}=\sigma$ :

$$
a m=\left(\ln P+\frac{a^{2} \sigma^{2}}{2}\right)+\ln \left(\sum_{j=0, j \neq b}^{B} e^{-\eta \ln r_{j}}\right)-\frac{a^{2} \sigma_{t}^{2}}{2}
$$

We can express the mean interference power $\overline{I_{e x t}}$ received by a mobile as:

$$
\ln \left(\overline{I_{e x t}}\right)=\left(\ln P+\frac{a^{2} \sigma^{2}}{2}\right)+\ln \left(\sum_{j=0, j \neq b}^{B} r_{j}^{-\eta}\right)-\frac{a^{2} \sigma_{t}^{2}}{2}
$$

The variance $a^{2} \sigma_{t}^{2}$ of the sum of interferences is written as

$$
a^{2} \sigma_{t}^{2}=\ln \left(\frac{\sum_{j} e^{\left(2 a m_{j}+a^{2} \sigma^{2}\right)\left(e^{a^{2} \sigma^{2}}-1\right)}}{\left(\sum_{j} e^{a m_{j}+\frac{a^{2} \sigma^{2}}{2}}\right)^{2}}+1\right)
$$

Introducing

$$
G(\eta)=\frac{\sum_{j=0, j \neq b}^{B} r_{j}^{-2 \eta}}{\left(\sum_{j=0, j \neq b}^{B} r_{j}^{-\eta}\right)^{2}}
$$

the mean value of the total interference received by a mobile is given by

$$
\overline{I_{e x t}}=P \sum_{j=0, j \neq b}^{B} r_{j}^{-\eta} e^{\frac{a^{2} \sigma^{2}}{2}}\left(\left(e^{a^{2} \sigma^{2}}-1\right) G(\eta)+1\right)^{-1 / 2}
$$

and

$$
a^{2} \sigma_{t}^{2}=\ln \left(\left(e^{a^{2} \sigma^{2}}-1\right) G(\eta)+1\right)+\ln \left(e^{a^{2} \sigma^{2}}\right)
$$

\section{APPENDIX 2: INTERFERENCE FACTOR}

Since the ratio of two lognormal RV's is also a lognormal $\mathrm{RV}$, the interference factor is also lognormally distributed with the following mean and logarithmic variance:

$$
m_{f}=\frac{\overline{I_{e x t}}}{\overline{I_{\text {int }}}}
$$

and thus, considering that: $P_{b}=P$, we can write, dropping the index b:

$$
m_{f}=\frac{\sum_{j} r_{j}^{-\eta}}{r^{-\eta}} e^{\frac{a^{2} \sigma^{2}}{2}}\left(\left(e^{a^{2} \sigma^{2}}-1\right) G(\eta)+1\right)^{-1 / 2}
$$

and finally, denoting:

$$
H(\sigma)=e^{\frac{a^{2} \sigma^{2}}{2}}\left(\left(e^{a^{2} \sigma^{2}}-1\right) G(\eta)+1\right)^{-1 / 2}
$$

we have

$$
m_{f}=f(\eta) e^{\frac{a^{2} \sigma^{2}}{2}}\left(\left(e^{a^{2} \sigma^{2}}-1\right) G(\eta)+1\right)^{-1 / 2}
$$

In a analogue analysis, the standard deviation is given by $a^{2} s_{f}^{2}=a^{2} \sigma_{t}^{2}+a^{2} \sigma^{2}$ so we have

$$
a^{2} s_{f}^{2}=2\left(a^{2} \sigma^{2}-\ln (H(\sigma))\right)
$$




\section{INTERFERENCE FACTOR FOR CORRELATED POWERS}

In [7], the coefficient $t_{k j}$ is introduced to take into account the correlation between the lognormal RV:

$$
t_{k j}=\frac{E\left[\left(X_{k}-m_{k}\right)\left(X_{j}-m_{j}\right)\right]}{\sigma_{k} \sigma_{j}} .
$$

where $X_{i}$ is a RV with mean $m_{i}$ and variance $\sigma_{i}^{2}$. The mean value $M_{\text {corr }}$ of the sum of $\mathrm{N}$ lognormal $\mathrm{RV}$ is thus calculated in [7] as follows.Denoting $u_{1}=\sum_{k=1}^{N} e^{\left(m_{k}+\frac{\sigma_{k}^{2}}{2}\right)}$ and

$$
\begin{aligned}
u_{2}= & \sum_{k=1}^{N} \exp \left(2 m_{k}+2 \sigma_{k}^{2}\right) \\
& +2 \sum_{k=1}^{N-1} \sum_{j=k+1}^{N} e^{\left(m_{k}+m_{j}\right)} e^{\frac{1}{2}\left(\sigma_{k}^{2}+\sigma_{j}^{2}+2 t_{k j} \sigma_{k} \sigma_{j}\right)}
\end{aligned}
$$

the mean value $M_{\text {corr }}$ of the sum of $N$ lognormal RV is thus calculated in [7] as:

$$
M_{\text {corr }}=2 \ln u_{1}-\frac{1}{2} \ln u_{2}=\ln u_{1}-\frac{1}{2} \frac{\ln u_{2}}{u_{1}^{2}}
$$

Let's consider that all the RV have the same variance $\sigma$. We moreover introduce a mean value of the correlation coefficient $t=\overline{t_{k j}}$. We can write

$$
\begin{array}{r}
2 \sum_{k=1}^{N-1} \sum_{j=k+1}^{N} e^{\left(m_{k}+m_{j}\right)} e^{\frac{1}{2}\left(\sigma_{k}^{2}+\sigma_{j}^{2}+2 t_{k j} \sigma_{k} \sigma_{j}\right)} \\
=2 \sum_{k=1}^{N-1} \sum_{j=k+1}^{N} e^{\left(m_{k}+m_{j}\right)} e^{\left(\sigma^{2}+t \sigma^{2}\right)} \\
=\left(\frac{1}{2} \sum_{j=1}^{N} e^{\left(m_{j}+\sigma^{2} / 2\right)}+\frac{1}{2} \sum_{k=1}^{N} e^{\left(m_{k}+\sigma^{2} / 2\right)}\right)^{2} e^{\left(t \sigma^{2}\right)} \\
-\frac{1}{2}\left(\sum_{j=1}^{N} e^{\left(2 m_{j}+\sigma^{2}\right)}+\sum_{k=1}^{N} e^{\left(2 m_{k}+\sigma^{2}\right)}\right) e^{\left(t \sigma^{2}\right)}
\end{array}
$$

so we have

$$
\begin{array}{r}
2 \sum_{k=1}^{N-1} \sum_{j=k+1}^{N} e^{\left(m_{k}+m_{j}\right)} e^{\left(\sigma^{2}+t \sigma^{2}\right)} \\
=\left(\sum_{j=1}^{N} e^{\left(m_{j}+\sigma^{2} / 2\right)}\right)^{2} e^{\left(t \sigma^{2}\right)}-\left(\sum_{k=1}^{N} e^{\left(2 m_{k}+\sigma^{2}\right)}\right) e^{\left(t \sigma^{2}\right)}
\end{array}
$$

so we have (from 42)

$$
\begin{aligned}
u_{2}= & \left(\sum_{k=1}^{N} e^{\left(2 m_{k}+\sigma^{2}\right)}\right) e^{\left(\sigma^{2}\right)}+\left(\sum_{k=1}^{N} e^{\left(m_{k}+\sigma^{2} / 2\right)}\right)^{2} e^{\left(t \sigma^{2}\right)} \\
& -\left(\sum_{k=1}^{N} e^{\left(2 m_{k}+\sigma^{2}\right)}\right) e\left(t \sigma^{2}\right)=\left(\sum_{k=1}^{N} e^{\left(m_{k}+\sigma^{2} / 2\right)}\right)^{2} e^{(t \sigma} \\
& +\left(\sum_{k=1}^{N} e^{\left(2 m_{k}+\sigma^{2}\right)}\right)\left(e^{\sigma^{2}}-e^{\left(t \sigma^{2}\right)}\right)
\end{aligned}
$$

so we express

$\ln \frac{u_{2}}{u_{1}^{2}}=\ln \left(\left(e^{\left(\sigma^{2}\right)}-e^{\left(t \sigma^{2}\right)}\right) \frac{\sum_{k=1}^{N} e^{2 m_{k}+\sigma^{2}}}{\left(\sum_{k=1}^{N} e^{m_{k}+\sigma^{2} / 2}\right)^{2}}+e^{\left(t \sigma^{2}\right)}\right)$

This expression is analogue to the one we established for the variance of the sum of interferences. So, taking into account a correlation between the RV, the parameter $a$, and using the expression of $M_{c o r r}$, the expression of the mean value of the interference factor becomes

$m_{f, \text { corr }}=\frac{\sum_{j} r_{j}^{-\eta}}{r^{-\eta}} e^{\left(\frac{a^{2} \sigma^{2}}{2}\right)}\left(\left(e^{a^{2} \sigma^{2}}-e^{t a^{2} \sigma^{2}}\right) G(\eta)+e^{t a^{2} \sigma^{2}}\right)^{-1 / 2}$

and finally denoting

$$
H_{\text {corr }}(\sigma)=e^{\left(\frac{a^{2} \sigma^{2}}{2}(1-t)\right)}\left(\left(e^{\left(a^{2} \sigma^{2}\right)(1-t)}-1\right) G(\eta)+1\right)^{-1 / 2}
$$

we can write, denoting $\sigma_{g}^{2}=\sigma^{2}(1-t)$

$$
m_{f, c o r r}=f(\eta) H_{c o r r}(\sigma)=f(\eta) H\left(\sigma_{g}\right) .
$$

And we have for the variance:

$$
a^{2} s_{f, c o r r}^{2}=a^{2} \sigma^{2}(1+t)+\ln \left(\left(e^{a^{2} \sigma^{2}(1-t)}-1\right) G(\eta)+1\right) .
$$

\section{REFERENCES}

[1] A. J. Viterbi, A. M. Viterbi, and E. Zehavi, Other-Cell Interference in Cellular Power-Controlled CDMA, IEEE Trans. on Communications, Vol. 42, No. 2/3/4, Freb/Mar/Apr. 1994.

[2] A. J. Viterbi, CDMA - Principles of Spread Spectrum Communication, Addison-Wesley, 1995.

[3] X. Lagrange, Principes et évolutions de l'UMTS, Hermes, 2005.

[4] L.Fenton "The sum of lognormal probability distributions in scatter transmission system", IEEE (IRE) Transactions on Communications, CS8,1960

[5] S. Shwartz and Y.S. Yeh, On the distributions functions and moments of power sums with lognormal components, Bell Syst, Tech J, vol 61, pp 1441 1462, Sept 1982

[6] G.L. Stuber, principles of Mobiles Communications, Norwell , MA. Kluwer, 1996

[7] Adnan A. Abu-Dayya and Norman C. Beaulieu, Outage Probabilities in the Presence of Correlated Lognormal Interferers, IEEE Transactions on Vehicular Technology, Vol. 43, N 1, February 1994

[8] J.-M. Kelif, Admission Control on Fluid CDMA Networks, Proc. of WiOpt, Apr. 2006.

[9] J-M. Kelif and E. Altman, Downlink Fluid Model of CDMA Networks, Proc. of IEEE VTC Spring, May 2005.

[10] J-M Kelif, M. Coupechoux and P. Godlewski, Spatial Outage Probability for Cellular Networks, Proc. of IEEE Globecom Washington, Nov. 2007

[11] F. Baccelli, B. Blaszczyszyn, and M. K. Karray, Blocking Rates in Large CDMA Networks via a Spatial Erlang Formula, Proc. of IEEE INFOCOM, Mar. 2005.

[12] C. C. Chan and Hanly, Calculating the Outage Probabability in CDMA Network with Spatial Poisson Traffic, IEEE Trans. on Vehicular Technology, Vol. 50, No. 1, Jan. 2001.

[13] S. E. Elayoubi and T. Chahed, Admission Control in the Downlink of WCDMA/UMTS, Lect. notes comput. sci., Springer, 2005.

[14] J. S. Evans and D. Everitt, Effective Bandwidth-Based Admission Control for Multiservice CDMA Cellular Networks, IEEE Trans. on Vehicular Technology, Vol. 48, No. 1, Jan. 1999.

15] J. M. Kelif, Modèle fluide pour les réseaux sans fils, Thése de doctorat, TELECOM ParisTech, 2008. 